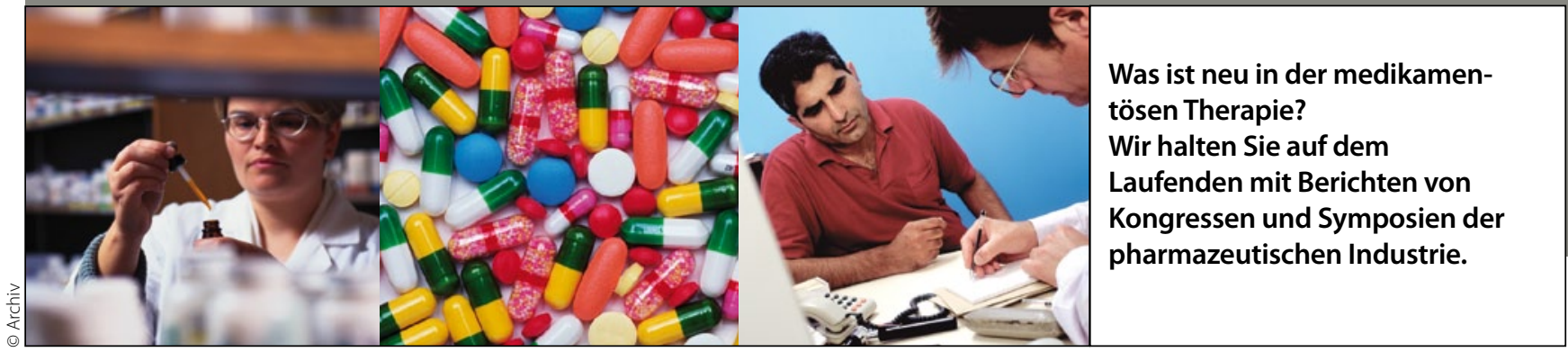

\title{
Vorhofflimmern
}

\section{Neue Therapieoption zur raschen Kardioversion}

- Zur medikamentösen Kardioversion bei Vorhofflimmern gibt es nur eine begrenzte Zahl von Optionen, von denen die älteren Substanzen nicht vernachlässigbare Nachteile wie proarrhythmisches Risiko, Kontraindikationen etwa bei strukturellen Herzerkrankungen sowie Interaktionen mit anderen Arzneimitteln aufweisen. Deshalb sollte primäres Auswahlkriterium die Sicherheit des Medikaments sein, so Prof. Paulus Kirchhof, Münster. Im EKG gebe es klare Hinweise, welcher Patient ein erhöhtes proarrhythmisches Risiko hat.

Mit Vernakalant (Brinavess ${ }^{\circledR}$ ) steht nun ein Antiarrhythmikum zur raschen Kardioversion bei erst seit kurzer Zeit bestehendem Vorhofflimmern zur Verfügung. In den vier zulassungsrelevanten klinischen Studien wurde Vernakalant bei hämodynamisch stabilen Patienten mit Placebo (drei Studien) und Amiodaron (eine Studie) verglichen. Die Patienten hatten symptomatisches Vorhofflimmern mit einer Dauer von wenigen Stunden bis maximal sieben Tagen. Endpunkt der Studien war der Prozentsatz der Patienten, die innerhalb von

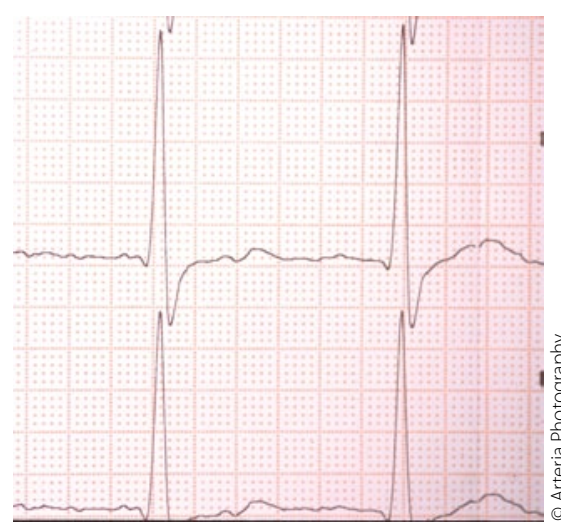

Proarrhythmisches Risiko bei Vorhofflimmern im EKG abklären.
90 Minuten nach Therapiebeginn im Sinusrhythmus waren. Mit intravenösem Vernakalant wurde dies bei etwa $50 \%$ erreicht, in der Placebogruppe bei weniger als 5\% ein signifikanter Unterschied.

Beim Vergleich von Vernakalant mit Amiodaron in der AVRO-Studie erreichten $51,7 \%$ der Patienten der Vernakalantgruppe wieder Sinusrhythmus, von den Amiodaronpatienten nur 5,2\%. An dieser Studie nahmen auch Patienten mit struktureller Herzerkrankung teil, für die Vernakalant bei hämodynamischer Stabilität eine neue Option ist. Der Mehrkanalblocker, der auch zur Konversion bei postoperativem Vorhofflimmern nach Herzoperation eingesetzt werden kann, verlängert selektiv im Vorhof die effektive Refraktärperiode. Zur Zeit wird Vernakalant auch zur oralen Applikation entwickelt.

\section{Wann kontraindiziert?}

Kontraindiziert ist das Antiarrhythmikum bei schwerer Aortenklappenstenose, Hypotension (unter $100 \mathrm{mmHg}$ systolisch), frischem Myokardinfarkt oder akutem Koronarsyndrom innerhalb der vergangenen 30 Tage und Herzinsuffizienz der Stadien NYHA III und IV, schwerer Bradykardie, Sinusknotendysfunktion oder AV-Block 2. oder 3. Grades ohne Schrittmacher, Patienten mit QT-Verlängerung (unkorrigiert $>440 \mathrm{~ms}$ ) zu Behandlungsbeginn sowie nach intravenöser Anwendung von Antiarrhythmika zur Rhythmuskontrolle (Klasse I und Klasse III) innerhalb von vier Stunden vor sowie innerhalb der ersten vier Stunden nach der Anwendung von Vernakalant. Häufigste Nebenwirkungen waren metallischer Geschmack, vermehrtes Niesen und Übelkeit.
Ob eine frühzeitige umfassende und standardisierte rhythmuserhaltende Therapie tatsächlich geeignet ist, die mit länger anhaltendem Vorhofflimmern verbundenen Komplikationen, einschließlich Schlaganfall und Tod, zu verhindern, soll jetzt in der prospektiven, randomisierten und multizentrischen Studie EAST (Early comprehensive Atrial fibrillation Stroke prevention Trial) untersucht werden, berichtete Kirchhof, Leiter der Studie. Dazu sollen mehr als 3000 Patienten mit neu aufgetretenem Vorhofflimmern und erhöhtem Schlaganfall- und Sterberisiko rekrutiert werden.

\section{- Dr. Monika Prinoth, Heidelberg}

Quelle: Satellitensymposium „New Advances for the Cardioversion of Recent Onset Atrial Fibrillation" ESC-Kongress, Paris, August 2011 (Veranstalter: MSD Sharp \& Dohme)

\section{Kurz notiert}

Obstipationstherapie $>$ Mit dem neuen $\mathrm{Movicol}^{\circledR}$ flüssig Orange ergänzt Norgine sein Sortiment um eine flüssige Darreichungsform. Diese lässt sich noch individueller dosieren. Zudem ist sie einfacher in der Handhabung: sie löst sich vollständig in Wasser auf. Damit entfällt das von vielen Anwendern als lästig empfundene lange Umrühren bei der Pulverformulierung sowie das Stauben bei der Zubereitung. Das flüssige Macrogol ist zugelassen zur Behandlung akuter und chronischer Obstipation bei Patienten ab zwölf Jahren und erhältlich in den Packungsgrößen 250 und $500 \mathrm{ml}$. Norgine 